\title{
Energy Decay and Asymptotic Behavior of Solutions to the Wave Equations with Linear Dissipation
}

\author{
Kiyoshi MochIZUKI * and Hideo NAKAZAWA *
}

\section{§1. Introduction}

Let $\Omega \subset \mathbf{R}^{N}$ be an unbounded domain with smooth boundary $\partial \Omega$. We consider the mixed initial-boundary value problem

$$
\begin{cases}w_{t t}-\Delta w+b(x, t) w_{t}=0, & (x, t) \in \Omega \times(0, \infty) \\ w(x, 0)=w_{1}(x), w_{t}(x, 0)=w_{2}(x), & x \in \Omega \\ w(x, t)=0, & (x, t) \in \partial \Omega \times(0, \infty)\end{cases}
$$

where $w_{t}=\partial w / \partial t, w_{t t}=\partial^{2} w / \partial t^{2}, \Delta$ is the $N$-dimensional Laplacian and $b(x, t)$ is a nonnegative $C^{1}$-function.

Let $H^{k}(\Omega), k=0,1,2, \cdots$, be the usual Sobolev space with norm

$$
\|f\|_{H^{k}}=\left\{\sum_{|\alpha| \leq k} \int_{\Omega}\left|\nabla^{\alpha} f(x)\right|^{2} d x\right\}^{1 / 2}<\infty
$$

where $\alpha$ are multiindices. We write $H^{0}(\Omega)=L^{2}(\Omega)$ and $\|f\|_{L^{2}}=\|f\| . \quad H_{0}^{1}(\Omega)$ is the completion in $H^{1}(\Omega)$ of the set of all smooth functions with compact support in $\Omega$. Let $E$ be the space of all pairs $f=\left\{f_{1}, f_{2}\right\}$ of functions such that

$$
\|f\|_{E}^{2}=\left\|\left\{f_{1}, f_{2}\right\}\right\|_{E}^{2}=\frac{1}{2}\left(\left\|f_{2}\right\|^{2}+\left\|\nabla f_{1}\right\|^{2}\right)<\infty .
$$

For solution $w(t)$ of (1.1), we simply write

$$
\|w(t)\|_{E}^{2}=\left\|\left\{w(t), w_{t}(t)\right\}\right\|_{E}^{2}
$$

and call it the energy of $w(t)$ at time $t$.

Communicated by T. Kawai, May 29, 1995.

1991 Mathematics Subject Classification (s): $35 \mathrm{~L}$

* Department of Mathematics, Tokyo Metropolitan University, Hachioji, Tokyo 192-03, Japan 
Now, assume

$$
\left\{w_{1}, w_{2}\right\} \in\left[H^{2}(\Omega) \cap H_{0}^{1}(\Omega)\right] \times H_{0}^{1}(\Omega) .
$$

Then as is well known, the initial-boundary value problem (1.1) has a global solution in the class

$$
w(\cdot, t) \in C^{0}\left([0, \infty) ; H^{2}(\Omega)\right) \cap C^{1}\left([0, \infty) ; H_{0}^{1}(\Omega)\right) \cap C^{2}\left([0, \infty) ; L^{2}(\Omega)\right) .
$$

Moreover, we have the energy equation

$$
\|w(t)\|_{E}^{2}+\int_{0}^{t} \int_{\Omega} b(x, \tau) w_{t}(x, \tau)^{2} d x d \tau=\|w(0)\|_{E}^{2}
$$

for any $t>0$.

Since $b(x, t) \geq 0, b(x, t) w_{t}$ represents a friction of viscous type, and we see from (1.4) that the energy $\|w(t)\|_{E}^{2}$ of solution $w(t)$ is decreasing in $t>0$. Thus, a question naturally rises whether it decays or not as $t$ goes to infinity.

The decay and nondecay problems have been studied in works of Matsumura [1] and Mochizuki [2], [3] in case where $\Omega=\mathbb{R}^{N}$. It is proved in [1] that the energy decays if $b_{0}(1+r+t)^{-1} \leq b(x, t) \leq b_{1} \quad(r=|x|)$ and $b_{t}(x, t)$ $\leq 0$. (Note that Matsumura's result is restricted to the compactly supported initial data. Its noncompact version is given in [3] .) On the other hand, it is proved in [2], [3] that if $0 \leq b(x, t) \leq b_{2}(1+r)^{-r}, \gamma>1$, then the energy does not in general decay and every solution with finite energy is asymptotically free as $t \rightarrow \infty$.

From these results we see that if $b(x, t)=O\left(r^{-\gamma}\right)$ as $r=|x| \rightarrow \infty$, then $\gamma=1$ is the critical exponent of energy decay. Our purpose of the present paper is to improve this result. We consider the case $b(x, t)=o\left(r^{-1}\right)$ and obtain the critical exponent of logarithmic order.

In order to state the assumption on $b(x, t)$, we define the positive number $e_{n}$ and the function $\log { }^{[n]}(n=0,1,2, \cdots)$ by

$$
\begin{gathered}
e_{0}=1, e_{1}=e, \cdots, e_{n}=e^{e_{n-1}}, \\
\log ^{[0]} a=a, \log ^{[1]} a=\log a, \cdots, \log ^{[n]} a=\log \log ^{[n-1]} a .
\end{gathered}
$$

In the following we require one of the following (A1) and (A2).

(A1) There exist $b_{0}, b_{1}>0$ and a nonnegative integer $n$ such that

$$
b_{0}\left\{\left(e_{n}+r+t\right) \log \left(e_{n}+r+t\right) \cdots \log ^{[n]}\left(e_{n}+r+t\right)\right\}^{-1} \leq b(x, t) \leq b_{1} .
$$


Moreover,

$$
b_{t}(x, t) \leq 0,(x, t) \in \Omega \times(0, \infty) .
$$

(A2) $N \geq 3$ and a $\mathbb{R}^{N} \backslash \Omega$ is starshaped with respect to the origin $x=0$. There exist $b_{2}>0, \gamma>1$ and a nonnegative integer $n$ such that

$$
0 \leq b(x, t) \leq b_{2}\left\{\left(e_{n}+r\right) \cdots \log ^{[n-1]}\left(e_{n}+r\right)\left[\log ^{[n]}\left(e_{n}+r\right)\right]^{r}\right\}^{-1}
$$

Our results on the energy decay are summarized in the following

Theorem 1. Assume (A1). Let $\left\{w_{1}, w_{2}\right\}$ satisfy (1.2) and

$$
\int_{\Omega} \log ^{[n]}\left(e_{n}+r\right)\left\{w_{2}^{2}+\left|\nabla w_{1}\right|^{2}\right\} d x<\infty .
$$

Then the energy of the solution to (1.1) decays as $t$ goes to infinity. More precisely, there exists a constant $K=K\left(w_{0}, w_{1}, n\right)>0$ such that

$$
\|w(t)\|_{E}^{2} \leq K\left\{\log ^{[n]}\left(e_{n}+t\right)\right\}^{-\mu},
$$

where $\mu=\min \left\{1, b_{0} / 2\right\}$.

To state another theorem, we need a local decay estimate for the free wave equation in $\Omega$ :

$$
\begin{cases}w_{0 t t}-\Delta w_{0}=0, & (x, t) \in \Omega \times(0, \infty) \\ w_{0}(x, 0)=f_{1}(x), w_{0 t}(x, 0)=f_{2}(x), & x \in \Omega \\ w(x, t)=0, & (x, t) \in \partial \Omega \times(0, \infty)\end{cases}
$$

As we shall show in Lemma 3.3, if $N$ and $\Omega$ satisfies the conditions in (A2), then we have

$$
\int_{0}^{\infty} \int_{\Omega}\left\{\left(e_{n}+r\right) \log \left(e_{n}+r\right) \cdots\left[\log ^{[n]}\left(e_{n}+r\right)\right]^{r}\right\}^{-1} w_{0 t}^{2} d x d t \leq C\|f\|_{E}^{2}
$$

for some $C>0$ independent of $f=\left\{f_{1}, f_{2}\right\} \in E$.

With this inequality, our results on energy nondecay and asymptotics are summarized in the following

Theorem 2. Assume (A2). (a) Let $f=\left\{f_{1}, f_{2}\right\} \in E$ and $w_{0}(t)$ be the solution to (1.7). We choose $\sigma>0$ to satisfy

$$
\int_{\sigma}^{\infty} \int_{\Omega}\left\{\left(e_{n}+r\right) \log \left(e_{n}+r\right) \cdots\left[\log ^{[n]}\left(e_{n}+r\right)\right]^{r}\right\}^{-1} w_{0 t}^{2} d x d t \leq 4 b_{2}^{-1}\|f\|_{E}^{2}
$$


Let $w_{\sigma}(t)$ be the solution to (1.1) with the initial data

$$
\left\{w_{\sigma}(0), w_{\sigma t}(0)\right\}=\left\{w_{0}(\sigma), w_{0 t}(\sigma)\right\}
$$

Then the energy of this solution remains positive as $t$ goes to infinity.

(b) For any solution $w(t)$ of (1.1) with $\left\{w_{1}, w_{2}\right\} \in E$, there exists a pair $f^{+}$ $=\left\{f_{1}^{+}, f_{2}^{+}\right\} \in E$ such that

$$
\left\|w(t)-w_{0}^{+}(t)\right\|_{E} \rightarrow 0 \quad \text { as } \quad t \rightarrow \infty
$$

where $w_{0}^{+}(t)$ is the solution to (1.7) with $f$ replaced by $f^{+}$.

Our argument on the decay property is based on a weighted energy inequality. So, the same results as Theorem 1 can be obtained also for the problem with Neumann or Robin boundary condition. On the other hand, to show Theorem 2 we combine the usual energy estimate and inequality (1.8). A similar treatment is found e.g., in [3].

In the case where $\Omega$ is bounded, there are many works on the energy decay. However, in the case of unbounded domain there are not so many works other than [1], [3]. We refer here Nakao [5] and Zuazua [7], where are treated the Klein-Gordon equations with dissipative term. As for the energy nondecay, another approach is developed in Rauch-Taylor [6] for $b(x, t)$ with compact support in $x$.

Theorems 1 and 2 are proved in $\S 2$ and $\S 3$, respectively. In $\S 4$ we remark that our proof of the energy decay can be applied to some quasilinear wave equations.

\section{§2. Proof of Theorem 1}

Let $\varphi(s), s \geq 0$, be a smooth function satisfying

$$
\varphi(s) \geq 1 \text { and } \lim _{s \rightarrow \infty} \varphi(s)=\infty ;
$$

$\varphi^{\prime}(s)>0, \varphi^{\prime \prime}(s) \leq 0, \varphi^{\prime \prime \prime}(s) \geq 0$ and they all are bounded in $s \geq 0$;

$$
2 \varphi^{\prime}(s) \varphi^{\prime \prime \prime}(s)-\varphi^{\prime \prime}(s)^{2} \geq 0
$$

With this $\varphi(s)$ we define a weighted energy of solutions at time $t$ as follows:

$$
\|w(t)\|_{E_{\varphi}}^{2}=\frac{1}{2} \int_{\Omega} \varphi(r+t)\left(w_{t}^{2}+|\nabla w|^{2}\right) d x,
$$


where $r=|x|$. In order to show a energy decay property, the initial data are required other than (1.2) to satisfy

$$
\|w(0)\|_{E_{\varphi}}<\infty
$$

(cf., (1.5)) .

Multiply by $\{\varphi(r+t) w\}_{t}$ on both sides of (1.1). It then follows that

$$
X_{t}+\nabla \cdot Y+Z=0
$$

where

$$
\begin{aligned}
X= & \frac{1}{2} \varphi\left\{w_{t}^{2}+|\nabla w|^{2}\right\}+\varphi^{\prime} w_{t} w+\frac{1}{2}\left(\varphi^{\prime} b-\varphi^{\prime \prime}\right) w^{2}, \\
Y= & -\left(\varphi w_{t}+\varphi^{\prime} w\right) \nabla w, \\
Z= & \left(\varphi b-2 \varphi^{\prime}\right) w_{t}^{2}+\frac{1}{2} \varphi^{\prime}\left|\frac{x}{r} w_{t}+\nabla w+\frac{x}{r} \varphi^{\prime-1} \varphi^{\prime \prime} w\right|^{2} \\
& +\frac{1}{2}\left\{\varphi^{\prime \prime \prime}-\varphi^{\prime-1} \varphi^{\prime \prime 2}-\left(\varphi^{\prime} b\right)_{t}\right\} w^{2}-\varphi^{\prime \prime} w_{t} w .
\end{aligned}
$$

Making use of the identity

$$
-\varphi^{\prime \prime} w_{t} w=-\frac{1}{2} \partial_{t}\left[\varphi^{\prime \prime} w^{2}\right]+\frac{1}{2} \varphi^{\prime \prime \prime} w^{2}
$$

and noting (2.3), we easily have

$$
Z \geq\left(\varphi b-2 \varphi^{\prime}\right) w_{t}^{2}-\frac{1}{2}\left(\varphi^{\prime} b\right)_{t} w^{2}-\frac{1}{2} \partial_{t}\left[\varphi^{\prime \prime} w^{2}\right]
$$

Lemma 2.1. For any $t>0$ and $0<\epsilon<1$, the solution $w(t)$ of (1.1) admits the inequality

$$
\begin{aligned}
& (1-\epsilon)\|w(t)\|_{E_{\varphi}}^{2}+\frac{1}{2} \int_{\Omega}\left(-2 \varphi^{\prime \prime}+\varphi^{\prime} b-\epsilon^{-1} \varphi^{-1} \varphi^{2}\right) w^{2} d x \\
& \quad+\int_{0}^{t} \int_{\Omega}\left\{\left(\varphi b-2 \varphi^{\prime}\right) w_{t}^{2}-\frac{1}{2}\left(\varphi^{\prime} b\right)_{t} w^{2}\right\} d x d \tau \\
& \leq(1+\epsilon)\|w(0)\|_{E_{\varphi}}^{2}+\frac{1}{2} \int_{\Omega}\left(-2 \varphi^{\prime \prime}+\varphi^{\prime} b+\epsilon^{-1} \varphi^{-1} \varphi^{\prime 2}\right) w_{1}^{2} d x .
\end{aligned}
$$

Proof. Let $\Omega(R)=\{x \in \Omega ;|x|<R\}$ and $S_{\Omega}(R)=\{x \in \Omega ;|x|=R\}$. We integrate (2.6) over $\Omega(R) \times(0, t)$. Then integration by parts and (2.7) give 


$$
\begin{gathered}
\left.\int_{\Omega(R)}\left\{X(x, \tau)-\frac{1}{2} \varphi^{\prime \prime}(r+\tau) w(x, \tau)^{2}\right\} d x\right|_{\tau=0} ^{\tau=t}+\int_{0}^{t} \int_{S_{\Omega}(R)} \frac{x}{r} \circ Y(x, \tau) d S d \tau \\
+\int_{0}^{t} \int_{\Omega(R)}\left\{\left(\varphi b-2 \varphi^{\prime}\right) w_{t}^{2}-\frac{1}{2}\left(\varphi^{\prime} b\right)_{t} w^{2}\right\} d x d \tau \leq 0
\end{gathered}
$$

By the Schwarz inequality

$$
\begin{aligned}
& X(x, t)-\frac{1}{2} \varphi^{\prime \prime}(r+t) w(x, t)^{2} \\
& \quad \geq \frac{1-\epsilon}{2} \varphi\left\{w_{t}^{2}+|\nabla w|^{2}\right\}+\frac{1}{2}\left(-2 \varphi^{\prime \prime}+\varphi^{\prime} b-\epsilon^{-1} \varphi^{-1} \varphi^{2}\right) w^{2}, \\
& X(x, 0)-\frac{1}{2} \varphi^{\prime \prime}(r) w(x, 0)^{2} \\
& \quad \leq \frac{1+\epsilon}{2} \varphi\left\{w_{2}^{2}+\left|\nabla w_{1}\right|^{2}\right\}+\frac{1}{2}\left(-2 \varphi^{\prime \prime}+\varphi^{\prime} b+\epsilon^{-1} \varphi^{-1} \varphi^{2}\right) w_{1}^{2} .
\end{aligned}
$$

Similarly, we have

$$
\left|\frac{x}{r} \cdot Y(x, \tau)\right| \leq \varphi\left(w_{t}^{2}+w_{r}^{2}\right)+\frac{1}{2} \varphi^{-1} \varphi^{\prime 2} w^{2}
$$

Note here (1.3), (2.2) and that $\varphi(s)=O(s)$ as $s \rightarrow \infty$. Then (2.12) implies

$$
\liminf _{R \rightarrow \infty} \int_{0}^{t} \int_{S_{\Omega}(R)}\left|\frac{x}{r} \cdot Y(x, \tau)\right| d S d \tau=0
$$

Thus, applying (2.10),(2.11) and letting $R \rightarrow \infty$ in (2.9), we conclude the assertion of the lemma.

Lemma 2.2. Let $w(t)$ be as in the above lemma. Suppose that

$$
\begin{aligned}
& \varphi(r+t) b(x, t) \geq 2 \varphi^{\prime}(r+t), \\
& \left\{\varphi^{\prime}(r+t) b(x, t)\right\}_{t} \leq 0
\end{aligned}
$$

for any $(x, t) \in \Omega \times(0, \infty)$. Then we have

$$
\text { (2.15) } \quad\|w(t)\|_{E_{\varphi}}^{2} \leq 3\|w(0)\|_{E_{\varphi}}^{2}+2 \int_{\Omega}\left\{-\varphi^{\prime \prime}(r)+\varphi^{\prime}(r) b(x, 0)\right\} w_{1}^{2}(x) d x<\infty
$$

Thus, the energy of $w(t)$ decays like 


$$
\|w(t)\|_{E}^{2}=O\left(\varphi(t)^{-1}\right) \text { as } t \rightarrow \infty
$$

Proof. We put $\epsilon=1 / 2$ in (2.8). Then it follows from (2.2) and (2.13) that

$$
-2 \varphi^{\prime \prime}+\varphi^{\prime} b-\epsilon^{-1} \varphi^{-1} \varphi^{2} \geq 0, \quad-2 \varphi^{\prime \prime}+\varphi^{\prime} b+\epsilon^{-1} \varphi^{-1} \varphi^{2} \leq 2\left(-\varphi^{\prime \prime}+\varphi^{\prime} b\right) .
$$

Applying these inequalities and (2.13), (2.14) in (2.8), we obtain (2.15) and hence (2.16).

Proof of Theorem 1. We choose

$$
\varphi(s)=\left[\log ^{[n]}\left(e_{n}+s\right)\right]^{\mu}
$$

Note that $\mu \leq 1$. Then (2.5) follows from condition (1.5). So, Theorem 1 is proved if we can verify that the above $\varphi$ satisfies conditions $(2.1) \sim(2.3)$ and (2.13), (2.14) of Lemma 2.2.

(2.1) is obvious from (2.17). Differentiating (2.17), we have

$$
\begin{aligned}
\varphi^{\prime}= & \mu[n]^{\mu-1}[n-1]^{-1} \cdots[2]^{-1}[1]^{-1}[0]^{-1}, \\
\varphi^{\prime \prime}= & -\mu[n]^{\mu-1}[n-1]^{-1} \cdots[2]^{-1}[1]^{-1}[0]^{-2} \\
& -\mu[n]^{\mu-1}[n-1]^{-1} . .[2]^{-1}[1]^{-2}[0]^{-2} \\
& \cdots \\
& -\mu[n]^{\mu-1}[n-1]^{-2} \ldots[2]^{-2}[1]^{-2}[0]^{-2} \\
& -\mu(1-\mu)[n]^{\mu-2}[n-1]^{-2} \cdots[2]^{-2}[1]^{-2}[0]^{-2}, \\
\varphi^{\prime \prime \prime}=\{- & \left.2 \sum_{i=0}^{n-1}[i]^{-1} \ldots[0]^{-1}-(2-\mu)[n]^{-1} \cdots[0]^{-1}\right\} \varphi^{\prime \prime} \\
- & \mu \sum_{k=1}^{n}[k]^{-1} \cdots[0]^{-1} \sum_{i=1}^{k}[n]^{\mu-1} \cdots[i]^{-1}[i-1]^{-2} \ldots[0]^{-2},
\end{aligned}
$$

where $[k]=\log ^{[k]}\left(e_{n}+s\right)(k=0,1 \cdots, n)$. These show (2.2) except the inequality $\varphi^{\prime \prime \prime}(s) \geq 0$, which also holds true since we have

$$
\begin{gathered}
\varphi^{\prime \prime \prime} \geq\left\{-2 \sum_{i=0}^{n-1}[i]^{-1} \cdots[0]^{-1}-(2-\mu)[n]^{-1} \cdots[0]^{-1}\right\} \varphi^{\prime \prime} \\
+\sum_{k=1}^{n}[k]^{-1} \cdots[0]^{-1} \varphi^{\prime \prime}
\end{gathered}
$$




$$
=\left\{-2[0]^{-1}-\sum_{k=1}^{n-1}[k]^{-1} \ldots[0]^{-1}-(1-\mu)[n]^{-1} . .[0]^{-1}\right\} \varphi^{\prime \prime}>0 .
$$

Next, note

$$
\begin{gathered}
\frac{\varphi^{\prime \prime}}{\varphi^{\prime}}=-\sum_{k=0}^{n-1}[k]^{-1} \cdots[0]^{-1}-(1-\mu)[n]^{-1} \cdots[0]^{-1} \\
\frac{\varphi^{\prime \prime \prime}}{\varphi^{\prime \prime}} \leq-2[0]^{-1}-\sum_{k=1}^{n-1}[k]^{-1} \cdots[0]^{-1}-(1-\mu)[n]^{-1} \cdots[0]^{-1}
\end{gathered}
$$

Then it follows that

$$
\frac{2 \varphi^{\prime \prime \prime}}{\varphi^{\prime \prime}}-\frac{\varphi^{\prime \prime}}{\varphi^{\prime}} \leq-3[0]^{-1}-\sum_{k=1}^{n-1}[k]^{-1} \cdots[0]^{-1}-(1-\mu)[n]^{-1} \cdots[0]^{-1} \leq 0 .
$$

This proves (2.3).

(2.13) easily follows from (A1), (2.17) and (2.18) since $\mu \leq b_{0} / 2$. (2.14) is obvious from (A1) and (2.2).

Thus, the assertion of Theorem 1 results from Lemma 2.2 if we choose

$$
K=3\|w(0)\|_{E_{\varphi}}^{2}+2 \int_{\Omega}\left\{-\varphi^{\prime \prime}(r)+\varphi^{\prime}(r) b(x, 0)\right\} w_{1}^{2}(x) d x
$$

in (2.15).

\section{§3. Proof of Theorem 2}

Throughout this $\S$ we assume (A2).

Let $\phi(s)$ be a positive smooth function of $s \geq 0$ satisfying

$$
\psi(s) \text { is bounded, monotone increasing in } s \text {, and } \phi(s) \geq s \psi^{\prime}(s) \text {. }
$$

We multiply by $\phi(r)\left(w_{r}+\frac{N-1}{2 r} w\right)$ on both sides of equation (1.1). It then follows that

$$
\widetilde{X}_{t}+\nabla \cdot \widetilde{Y}+\widetilde{Z}=0
$$

where 


$$
\begin{aligned}
\widetilde{X}= & \phi w_{t}\left(w_{r}+\frac{N-1}{2 r} w\right) \\
\widetilde{Y}= & -\frac{1}{2} \phi\left\{\frac{x}{r}\left(w_{t}^{2}-|\nabla w|^{2}+\frac{N-1}{2 r} w^{2}\right)+2 \nabla w\left(w_{r}+\frac{N-1}{2 r} w\right)\right\} \\
\widetilde{Z}= & \phi b w_{t}\left(w_{r}+\frac{N-1}{2 r} w\right) \\
& +\left(r^{-1} \psi^{\prime}-\psi^{\prime}\right)\left\{|\nabla w|^{2}-w_{r}^{2}+\frac{(N-1)(N-3)}{4 r^{2}} w^{2}\right\} \\
& +\frac{1}{2} \psi^{\prime}\left\{w_{t}^{2}+\left|\nabla w+\frac{N-1}{2 r} \frac{x}{r} w\right|^{2}+\frac{(N-1)(N-3)}{4 r^{2}} w^{2}\right\} .
\end{aligned}
$$

Lemma 3.1. Let $w(t)$ be the solution to (1.1) with finite energy. Then

$$
\begin{array}{r}
\frac{1}{2} \int_{0}^{t} \int_{\Omega} \phi^{\prime}\left\{w_{t}^{2}+\left|\nabla w+\frac{N-1}{2 r} \frac{x}{r} w\right|^{2}+\frac{(N-1)(N-3)}{4 r^{2}} w^{2}\right\} d x d \tau \\
\quad+\int_{0}^{t} \int_{\Omega} \phi b w_{t}\left(w_{r}+\frac{N-1}{2 r} w\right) d x d \tau \leq 2 \sup _{t>0} \int_{\Omega}|\widetilde{X}(x, t)| d x
\end{array}
$$

Proof. Integrate by parts the both sides of (3.2) over $\Omega \times(0, t)$. Then since $N \geq 3$ and $r^{-1} \phi-\phi^{\prime} \geq 0$, we have

$$
\begin{gathered}
\left.\int_{\Omega} \tilde{X} d x\right|_{\tau=0} ^{\tau=t}+\int_{0}^{t} \int_{\partial \Omega} \nu \cdot \widetilde{Y} d S d \tau+\int_{0}^{t} \int_{\Omega} \phi b w_{t}\left(w_{r}+\frac{N-1}{2 r} w\right) d x d \tau \\
+\frac{1}{2} \int_{0}^{t} \int_{\Omega} \phi^{\prime}\left\{w_{t}^{2}+\left|\nabla w+\frac{N-1}{2 r} \frac{x}{r} w\right|^{2}+\frac{(N-1)(N-3)}{4 r^{2}} w^{2}\right\} d x d \tau \leq 0
\end{gathered}
$$

where $\nu$ is the outer unit normal to the boundary $\partial \Omega$. By means of the boundary condition $\left.w\right|_{\partial \Omega}=0$,

$$
\begin{aligned}
\int_{0}^{t} \int_{\partial \Omega} \nu \cdot \tilde{Y} d S d \tau & =\frac{1}{2} \int_{0}^{t} \int_{\partial \Omega} \psi\left\{\left(\nu \cdot \frac{x}{r}\right)|\nabla w|^{2}-2(\nu \cdot \nabla w)\left(\frac{x}{r} \cdot \nabla w\right)\right\} d S d \tau \\
& =-\frac{1}{2} \int_{0}^{t} \int_{\partial \Omega} \psi\left(\nu \cdot \frac{x}{r}\right)|\nu \cdot \nabla w|^{2} d S d \tau .
\end{aligned}
$$

Here we have $(\nu \cdot x / r) \leq 0$ since the origin $\mathbf{R}^{N} \backslash \Omega$ is starshaped with respect to the origin. Thus, (3.3) holds.

Lemma 3.2. There exists a $C_{\psi}>0$ such that

$$
\int_{\Omega}|\tilde{X}(x, t)| d x \leq C_{\psi}\|w(0)\|_{E}^{2} \text { for any } t \geq 0
$$


Proof. By the Schwarz inequality we have

$$
\int_{\Omega}|\widetilde{X}(x, t)| d x \leq \sup _{s>0} \psi(s) \int_{\Omega}\left(w_{t}^{2}+\left|w_{r}+\frac{N-1}{2 r} w\right|^{2}\right) d x .
$$

Thus, (3.4) follows if we use the well known inequality

$$
\frac{(N-2)^{2}}{4} \int_{\Omega} \frac{1}{r^{2}} w^{2} d x \leq \int_{\Omega} w_{r}^{2} d x
$$

and (1.4).

Lemma 3.3. Let $w_{0}(t)$ be the solution to (1.7) with finite energy. Then

$$
\int_{0}^{\infty} \int_{\Omega}\left\{\left(e_{n}+r\right) \cdots \cdot \log ^{[n-1]}\left(e_{n}+r\right)\left[\log ^{[n]}\left(e_{n}+r\right)\right]^{r}\right\}^{-1} w_{0 t}^{2} d x d t \leq C\|f\|_{E}^{2}
$$

where $\gamma>1$ and $C=C(n, \gamma)$ is a positive constant independent of $w(t)$.

Proof. We put

$$
\phi(r)=1-\alpha\left\{\log ^{[n]}\left(e_{n}+r\right)\right\}^{-r+1}
$$

where $0<\alpha \leq \gamma^{-1}<1$. Then

$$
\psi^{\prime}(r)=\alpha(\gamma-1)\left\{\left(e_{n}+r\right) \cdots \log ^{[n-1 \mathrm{j}}\left(e_{n}+r\right)\left[\log ^{[n]}\left(e_{n}+r\right)\right]^{r}\right\}^{-1}
$$

and it follows that

$$
r^{-1} \phi(r) \geq(1-\alpha)\left(e_{n}+r\right)^{-1} \geq \alpha(\gamma-1)\left(e_{n}+r\right)^{-1} \geq \psi^{\prime}(r)
$$

Thus, (3.1) is satisfied for this $\psi(r)$.

We apply Lemmas 3.1 and 3.2 with this $\phi$ to the free solution $w_{0}(t)$. Then noting $b(x, t) \equiv 0$, we have

$$
\int_{0}^{t} \int_{\Omega} \psi^{\prime} w_{0 t}^{2} d x d \tau \leq 4 C_{\psi}\left\|w_{0}(0)\right\|_{E}^{2}
$$

Since $w_{0}(0)=f$, this and (3.6) show (3.5).

Our proof of Theorem 2 is based on Lemma 3.3 and the following usual energy equation. 
Lemma 3.4. We have

$$
2\left(w(t), w_{0}(t)\right)_{E}+\int_{0}^{t} \int_{\Omega} b(x, t) w_{t} w_{0 t} d x d \tau=2\left(w(0), w_{0}(0)\right)_{E}
$$

for any $t>0$, where

$$
2\left(w(t), w_{0}(t)\right)_{E}=\int_{\Omega}\left\{w_{t} w_{0 t}+\nabla w \cdot \nabla w_{0}\right\} d x
$$

Proof. Differentiate (3.8) and use equations (1.1) and (1.7). Then integrations by parts give

$$
2 \partial_{t}\left(w(t), w_{0}(t)\right)_{E}=-\int_{\Omega} b(x, t) w_{t} w_{0 t} d x
$$

Thus, integrating both sides over $(0, t)$, we obtain (3.7).

Proof of Theorem 2(a). For the solution $w_{0}(t)$ of $(1.7), w_{0}(t+\sigma)$ also satisfies (1.7) with $\left\{f_{1}, f_{2}\right\}$ replaced by $\left\{w_{0}(\sigma), w_{0 t}(\sigma)\right\}$. So, it follows from (1.10) and (3.7) that

$$
2\left(w_{\sigma}(t), w_{0}(t+\sigma)\right)_{E}+\int_{0}^{t} \int_{\Omega} b(x, \tau) w_{\sigma t}(\tau) w_{0 t}(\tau+\sigma) d x d \tau=2\left\|w_{0}(\sigma)\right\|_{E}^{2}
$$

Contrary to the conclusion, assume that $\left\|w_{\sigma}(t)\right\|_{E} \rightarrow 0$ as $t \rightarrow \infty$. Then since $\left\|w_{0}(t)\right\|_{E}$ is independent of $t$, letting $t \rightarrow \infty$ in (3.9), we obtain

$$
\int_{0}^{\infty} \int_{\Omega} b(x, t) w_{\sigma}(t) w_{0}(t+\sigma) d x d t=2\left\|w_{0}(\sigma)\right\|_{E}^{2}
$$

Thus, by the Schwarz inequality and (1.4),

$$
\int_{0}^{\infty} \int_{\Omega} b(x, t) w_{0}(t+\sigma)^{2} d x d t \geq 4\left\|w_{0}(\sigma)\right\|_{E}^{2}
$$

Since $\left\|w_{0}(\sigma)\right\|_{E}=\|f\|_{E}$, this contradicts to (1.9) under our reqirement (A2) on $b(x, t)$.

Theorem 2 (a) is thus proved.

Proof of Theorem $2(\mathrm{~b})$. Let $U_{0}(t), t \in \mathbb{R}$, be the unitary operator in the energy space $E$ which represents the solution $w_{0}(t)$ to $(1.7)$ : 


$$
\left\{w_{0}(t), w_{0 t}(t)\right\}=U_{0}(t) f
$$

Then it follows from (3.7) that

$$
\left(U_{0}(-t) w(t)-U_{0}(-s) w(s), f\right)_{E}=-\int_{s}^{t} \int_{\Omega} b(x, t) w_{t} w_{0 t} d x d \tau
$$

for any $0 \leq s<t$, where $w(t)$ stands for the pair $\left\{w(t), w_{t}(t)\right\}$. By the Schwarz inequality and (3.5) we have

$$
\left|\left(U_{0}(-t) w(t)-U_{0}(-s) w(s), f\right)_{E}\right| \leq C\left\{\int_{s}^{t} \int_{\Omega} b(x, t) w_{t}^{2} d x d \tau\right\}^{1 / 2}\|f\|_{E}
$$

$f=\left\{f_{1}, f_{2}\right\}$ being any pair in $E$, we see from (3.11) that

$$
\left\|U_{0}(-t) w(t)-U_{0}(-s) w(s)\right\|_{E} \rightarrow 0 \quad \text { as } \quad s, t \rightarrow \infty,
$$

and $U_{0}(-t) w(t)$ converges in $E$ as $t \rightarrow \infty$. Put

$$
f^{+} \equiv\left\{f_{1}^{+}, f_{2}^{+}\right\}=s-\lim _{t \rightarrow \infty} U_{0}(-t) w(t)
$$

Then $f^{+} \in E$ and we have

$$
\left\|w(t)-U_{0}(t) f^{+}\right\|_{E}=\left\|U_{0}(-t) w(t)-f^{+}\right\|_{E} \rightarrow 0 \quad \text { as } \quad t \rightarrow \infty .
$$

Theorem 2 (b) is thus proved.

\section{\$4. Energy Decay for Quasilinear Wave Equations}

In this $\S$ we remark that our proof of the energy decay can be applied to some quasilinear wave equations.

Consider the Cauchy problem

$$
\begin{cases}w_{t t}-\nabla \cdot\left\{\sigma\left(|\nabla w|^{2}\right) \nabla w\right\}+b(x, t) w_{t}=0, & (x, t) \in \mathbb{R}^{N}(0, \infty) \\ w(x, 0)=w_{1}(x), w_{t}(x, 0)=w_{2}(x) & x \in \mathbb{R}^{N},\end{cases}
$$

where $\sigma(s)=1 / \sqrt{1+s}$ and $b(x, t) \geq 0$. For the sake of simplicity, we assume

$$
\left\{w_{1}(x), w_{2}(x)\right\} \in C_{0}^{\infty}\left(\mathbb{R}^{N}\right) \times C_{0}^{\infty}\left(\mathbb{R}^{N}\right)
$$

The energy of solutions at time $t$ is defined by 


$$
\|w(t)\|_{E}^{2}=\frac{1}{2} \int_{\mathbf{R}^{N}}\left\{w_{t}(t)^{2}+\sigma_{1}\left(|\nabla w(t)|^{2}\right)\right\} d x
$$

and a weighted energy of solutions at time $t$ is defined by

$$
\|w(t)\|_{E_{\varphi}}^{2}=\frac{1}{2} \int_{\mathbf{R}^{N}} \varphi(r+t)\left\{w_{t}^{2}+\sigma_{1}\left(|\nabla w|^{2}\right)\right\} d x
$$

where

$$
\sigma_{1}(\eta)=\int_{0}^{\eta} \sigma(s) d s
$$

and $\varphi(s)$ is a function satisfying $(2.1) \sim(2.3)$.

Lemma 4.1. For any $t>0$ and $0<\epsilon<1$, the solution $w(t)$ of (4.1) admits the inequality

$$
\begin{aligned}
(1-\epsilon) & \left\|_{w}(t)\right\|_{E_{\varphi}}^{2}+\frac{1}{2} \int_{\mathbf{R}^{N}}\left(-2 \varphi^{\prime \prime}+\varphi^{\prime} b-\epsilon^{-1} \varphi^{-1} \varphi^{2}\right) w^{2} d x \\
& +\int_{0}^{t} \int_{\mathbf{R}^{N}}\left\{\left(\varphi b-2 \varphi^{\prime}\right) w_{t}^{2}-\frac{1}{2}\left(\varphi^{\prime} b\right){ }_{t} w^{2}\right\} d x d \tau \\
\leq & (1+\epsilon)\left\|_{w}(0)\right\|_{E_{\varphi}}^{2}+\frac{1}{2} \int_{\mathbf{R}^{N}}\left(-2 \varphi^{\prime \prime}+\varphi^{\prime} b+\epsilon^{-1} \varphi^{-1} \varphi^{\prime 2}\right) w_{1}^{2} d x .
\end{aligned}
$$

Proof. Multiply by $\{\varphi(r+t) w\}_{t}$ on both sides of (4.1). Then as in $\S 2$, it follows that

$$
X_{t}+\nabla \cdot Y+Z=0
$$

where

$$
\begin{aligned}
X= & \frac{1}{2} \varphi\left\{w_{t}^{2}+\sigma_{1}\left(|\nabla w|^{2}\right)\right\}+\varphi^{\prime} w_{t} w+\frac{1}{2}\left(\varphi^{\prime} b-\varphi^{\prime \prime}\right) w^{2} \\
Y= & -\left(\varphi w_{t}+\varphi^{\prime} w\right) \sigma\left(|\nabla w|^{2}\right) \nabla w \\
Z= & \left(\varphi b-2 \varphi^{\prime}\right) w_{t}^{2}+\frac{1}{2} \varphi^{\prime}\left|w_{t}+\sigma\left(|\nabla w|^{2}\right) w_{r}+\varphi^{\prime-1} \varphi^{\prime \prime} w\right|^{2} \\
& +\frac{1}{2} \varphi^{\prime}\left\{-\sigma_{1}\left(|\nabla w|^{2}\right)+2 \sigma\left(|\nabla w|^{2}\right)|\nabla w|^{2}-\sigma\left(|\nabla w|^{2}\right)^{2} w_{r}^{2}\right\} \\
& +\frac{1}{2}\left\{\varphi^{\prime \prime \prime}-\varphi^{\prime-1} \varphi^{\prime 2}-\left(\varphi^{\prime} b\right)_{t}\right\} w^{2}-\varphi^{\prime \prime} w_{t} w .
\end{aligned}
$$

Since we have 


$$
\begin{gathered}
-\sigma_{1}(s)+2 \sigma(s) s-\sigma(s)^{2} s=\left(1-\frac{1}{\sqrt{1+s}}\right)^{2} \geq 0 \\
-\varphi^{\prime \prime} w_{t} w=-\frac{1}{2} \partial_{t}\left[\varphi^{\prime \prime} w^{2}\right]+\frac{1}{2} \varphi^{\prime \prime \prime} w^{2},
\end{gathered}
$$

it follows that

$$
Z \geq\left(\varphi b-2 \varphi^{\prime}\right) w_{t}^{2}-\frac{1}{2}\left(\varphi^{\prime} b\right)_{t} w^{2}-\frac{1}{2} \partial_{t}\left[\varphi^{\prime \prime} w^{2}\right]
$$

Integrate by parts (4.4) over $\mathbb{R}^{N} \times(0, t)$. Then since $w(t)$ has a finite propagation speed, noting (4.2) and (4.5), we can follow the proof of Lemma 2.1 to conclude the assertion.

As in $\S 2$, we can easily prove the following theorem with this lemma.

Theorem 3. Assume (A1) with $\Omega=\mathbb{R}^{N}$, let $\left\{w_{1}, w_{2}\right\}$ satisfy (4.2) and let $w(t)$ be the corresponding solution to (4.1). If $w(t)$ is global, then its energy decays as $t$ goes to infinity. More precisely, there exists a $K=K\left(w_{0}, w_{1}, n\right)>0$ such that

$$
\|w(t)\|_{E}^{2} \leq K\left\{\log ^{[n]}\left(e_{n}+t\right)\right\}^{-\mu},
$$

where $\mu=\min \left\{1, b_{0} / 2\right\}$.

Remark. A similar result on the energy decay can be obtained for equations with nonlinear dissipation $b(x, t)\left|w_{t}\right|^{\rho-1} w_{t}$ under suitable restrictions on $b$ $(x, t)$ and $\rho>1$ as given in [4], where is studied decay and nondecay properties for semilinear equations.

\section{References}

[1] Matsumura, A., Energy decay of solutions of dissipative wave equations, Proc. Japan Acad., $\mathbf{5 3}$ (1977), 232-236.

[2] Mochizuki, K., Scattering theory for wave equations with dissipative terms, Publ. RIMS Kyoto Univ., 12 (1976), 383-390.

[3] Scattering Theory for Wave Equations (Japanese), Kinokuniya, 1984.

[4] Mochizuki, K. and Motai, T., On energy decay and nondecay problems for wave equations with nonlinear dissipative term in $\mathbb{R}_{N}$, J. Math. Soc. Japan, 47 (1995),405-421.

[5] Nakao, M., Energy decay of the wave equation with a nonlinear dissipative term, Funkcialaj Ekvacioj, 26 (1983), 237-250.

[6] Rauch, J. and Taylor, M., Decaying states of perturbed wave equation, J. Math. Anal. Appl., 54 (1976), 279-285.

[7] Zuazua, E., Exponential decay for the semilinear wave equations with localized damping in unbounded domains, J. Math. Pures Appl., 70 (1991), 513-529. 